\title{
Novel Approaches to avoid Microbial Adhesion onto Biomaterials
}

\section{Lígia R. Rodrigues}

IBB - Institute for Biotechnology and Bioengineering, Centre of Biological Engineering, University of Minho, Campus de Gualtar, 4710-057 Braga, Portugal

Infections resulting from microbial adhesion to biomaterial surfaces have been observed on nearly all medical devices with severe economic and medical consequences [1]. Biofilm infections, mainly due to their antimicrobial resistance, pose a number of clinical challenges, including disease, chronic inflammation, and rapidly acquired antibiotic resistance. Independently of the superiority of the implant, virtually all medical devices are prone to microbial colonization and infection. Examples of such devices include prosthetic heart valves, orthopaedic implants, intravascular catheters, artificial hearts, left ventricular assist devices, cardiac pacemakers, vascular prostheses, cerebrospinal fluid shunts, urinary catheters, voice prostheses, ocular prostheses and contact lenses, and intrauterine contraceptive devices.

A large amount of research to eliminate or reduce infections by developing anti-infective and anti-adhesive devices has been encouraged as a result of the significant resistance of biofilms to conventional antibiotic therapies. These improved devices may be produced by either mechanical design alternatives; physicochemical modification of the biomaterial surface (e.g. biosurfactants, plasma, atom transfer radical polymerization, brushes); anti-infective agents bound to the surface of the material (e.g. biosurfactants, silver, quaternary ammonium compounds, synthetic antibiotics); or release of toxic agents into the adjacent surroundings (e.g. chlorhexidine, antibiotics) [2,3]. The success of the mechanical design alternatives has been residual and with limited applicability [2]. Furthermore, the effectiveness of coatings designed to reduce adhesion by modification of the surface properties has also been reduced and greatly dependent on the bacterial species. Surfaces modified with poly(ethylene glycol) [4], poly(ethylene oxide) brushes [5], and hydrophilic polyurethanes [6], among many others, have been reported. Additionally, surface-bounded anti-infective agents are only toxic to the initial wave of incoming bacteria and provide little residual effects once layers of dead cells accumulate, which are also inflammatory [2].

On the other hand, a number of studies demonstrated some success in retarding microbial adhesion, and consequently inhibiting or delaying biofilm formation. The development of anti-adhesive silicone rubber surfaces for voice prostheses constitutes a good example. Since voice prostheses are continuously exposed to saliva, food, and drinks, together with the commensal microflora, they frequently fail and need to be replaced. Therefore, improvement of the antifouling properties of the silicone rubber material is highly desirable. Different approaches have been undertaken to modify the silicone rubber surface as an obvious strategy to inhibit biofilm formation and accordingly to prolong the lifetime of voice prostheses as reviewed by Rodrigues and collaborators [7]. An alternative approach is the design of coatings that actively release antibacterial agents with high initial fluxes during the first hours post-implementation. Nevertheless, it is desirable that release continues beyond this short term since fibrous capsule formation and tissue integration occur over longer periods of time [8]. A recent review on the development of antibiotic-releasing biomaterials has been compiled by Zilberman and Elsner [9].

Nonetheless, regardless of the method used to release an antibiotic from a biomaterial; this action is limited and will end as soon as the agent is depleted. Moreover, it is well known that the delivery of sub-lethal doses of antibiotics can lead to accelerated biofilm formation and induced virulence factor expression [2]. Given that the success the above mentioned strategies has been modest, mainly as a result of the various environments into which devices are placed and the diversity of ways in which organisms can colonize surfaces, the development of novel approaches to avoid microbial adhesion onto biomaterials, alternative to the traditional surface-modifying preventive ones, is an increasing challenge.

Biosurfactants represent an interesting approach because it may be possible to modify the surface properties to make it simultaneously anti-adhesive and give it antimicrobial activity. These microbial compounds are amphiphilic molecules with both hydrophilic and hydrophobic moieties and with a distinct tendency to accumulate at interfaces, thus affecting the adhesion and/or detachment of bacteria. Most interfaces have an overall negative or, hardly ever, positive charge. Gottenbos and co-workers [10] showed that positively charged biomaterial surfaces exert an antimicrobial effect on adhering Gram-negative, but not on Gram-positive bacteria.

Microbial surfactants constitute a diverse group of surface-active molecules and are known to occur in a variety of chemical structures, such as glycolipids, lipopeptides, fatty acids, neutral lipids, phospholipids, and polymeric structures [11]. The use and potential commercial application in the medical field of these compounds has increased during the past decade [11-13]. Due to their antibacterial, antifungal and antiviral activities, biosurfactants are useful for combating many diseases and as therapeutic agents. Furthermore, their role as anti-adhesive agents against several pathogens suggests their utility as suitable anti-adhesive coating agents for biomaterials, leading to new and effective means of combating colonization by pathogenic microorganisms without the use of synthetic drugs and chemicals [11].

Many researchers evaluated the potential of different biosurfactants as anti-adhesive coatings. For instance, Mireles and collaborators [14] pre-coated vinyl urethral catheters by running the surfactin solution through them before inoculation with media. As a result, it was found a decrease in the amount of biofilm formed by Salmonella typhimurium, Salmonella enterica, Escherichia coli and Proteus mirabilis. Also, probiotic lactobacilli were tested for the prevention of urogenital infections $[15,16]$. Other examples of the anti-adhesive effect of biosurfactants from lactobacilli against uropathogens include the work by Velraeds et al. [17] and by Heinemann et al. [18]. Additionally, biosurfactants from lactic acid bacteria, namely Streptococcus thermophilus $A$ and Lactococcus lactis 53, have been used as a strategy to avoid microbial colonization of silicone rubber voice prostheses [19,20]. Although a less pronounced anti-adhesive effect was found for yeast strains, over $90 \%$

*Corresponding author: Lígia R. Rodrigues, Department of Biological Engineering, University of Minho Campus, Portugal; E-mail: Irmr@deb.uminho.pt

Received 16 July, 2011; Accepted 16 July, 2011; Published 18 July, 2011

Citation: Rodrigues LR (2011) Novel Approaches to avoid Microbial Adhesion onto Biomaterials. J Biotechnol Biomaterial 1:104e. doi:10.4172/2155-952X.1000104e

Copyright: ( 2011 Rodrigues LR. This is an open-access article distributed under the terms of the Creative Commons Attribution License, which permits unrestricted use, distribution, and reproduction in any medium, provided the original author and source are credited. 
reductions in the initial deposition rates were achieved for most of the bacterial strains studied, being these strains responsible for prostheses failure. Furthermore, the biosurfactants produced by these lactic acid bacteria [21] and by Lactobacillus paracasei ssp. paracasei A20 [22] were found to possess antimicrobial and anti-adhesive activity against several Gram-positive and Gram-negative bacteria and filamentous fungi. The biosurfactant produced by L. paracasei A20 showed anti-adhesive activity against Streptococcus sanguis (72.9\%), S. aureus (76.8\%), S. epidermidis (72.9\%) and Streptococcus agalactie (66.6\%). These interesting findings constitute a promise regarding the potential use of biosurfactants against the adhesion of microorganisms responsible for diseases and infections in the urinary, vaginal and gastrointestinal tracts, and in the skin.

As discussed above, biosurfactants can play an important role in the development of anti-adhesive coatings for silicone rubber or other biomaterials as they effectively inhibit bacterial adhesion and retard biofilm formation. Therefore, surface and bulk modification techniques, laser-induced surface grafting and the sequential method for interpenetrating polymer networks should be explored as ways to link the biosurfactants more strongly with the biomaterial surfaces, thus avoiding their washout from the surfaces and prolonging their effect. In addition, being a suitable alternative to antimicrobial agents, biosurfactants can be used as safe and effective therapeutic agents. This is currently of major interest due to the concerns related with the increasing number of drug-resistant microorganisms and the need for alternative therapies [1]. Nevertheless, although biosurfactants constitute an interesting alternative to their chemical counterparts, mainly due to their biodegradability and low toxicity, it is important to stress that the insufficient data on their toxicity for humans, as well as their production costs, have been restraining their use in most applications.

The high cost of large-scale production is clearly a constraint for the commercialization of biosurfactants. Many biotechnological strategies have been pursued to reduce the production costs including the use of agro-industrial wastes or others as substrates, optimization of medium and culture conditions and efficient recovery processes [23]. However, the improvements obtained from these strategies are marginal and to successfully compete with synthetic surfactants, novel microorganisms must be designed. The use of hyper-producer strains allows increasing the production yields and consequently reducing costs. These strains can be screened from the natural environment, or engineered using synthetic biology approaches. Hence, data on the genes involved on the production of biosurfactants is critical for designing organisms with improved features. Once the genes have been indentified and isolated, they can be expressed in other microorganisms (e.g. to prevent pathogenicity), or they can be modified or placed under regulation of strong promoters to increase their expression and so enhance production. This knowledge will also allow the production of novel biosurfactants with specific new properties (designed by metabolic engineering and synthetic biology approaches) for different industrial applications. Further advances in genetic engineering of the known biosurfactant molecules could yield potent biosurfactants with altered antimicrobial profiles and decreased toxicity against mammalian cells.

In conclusion, it is fair to say that there is still no absolute solution for dealing with microbial adhesion onto medical devices, although biosurfactants seem to be potentially useful as a new generation of antiadhesive and antimicrobial coatings for such devices. Endeavors and challenges related with the genetics and structure-function relationships of biosurfactants, as well as the methods of binding them to surfaces, will continue to drive research in this field in the coming years.

\section{References}

1. Rodrigues LR (2011) Inhibition of bacterial adhesion on medical devices. In: Line D, and Goldman A (eds) Bacterial adhesion: biology, chemistry, and physics, Book Series "Advances in Experimental Medicine and Biology". Springer 715: $351-367$

2. Bryers JD (2008) Medical biofilms. Biotechnol Bioeng 100: 1-18.

3. Hetrick EM, Schoenfisch MH (2006) Reducing implant-related infections: active release strategies. Chem Soc Rev 35: 780-789.

4. Kingshott P, Wei J, Bagge-Ravn D, Gadegaard N, Gram L (2003) Covalent attachment of poly (ethylene glycol) to surfaces, critical for reducing bacterial adhesion. Langmuir 19: 6912-6921.

5. Kaper HJ, Busscher HJ, Norde W (2003) Characterization of poly (ethylene oxide) brushes on glass surfaces and adhesion of Staphylococcus epidermidis. J Biomater Sci Polym Ed 14: 313-24.

6. Berndt E, Behnke S, Dannehl A, Gajda A, Wingender J, et al. (2010) Functiona coatings for anti-biofouling applications by surface segregation of block copolymer additives. Polymer 51: 5910-5920.

7. Rodrigues LR, Banat IM, Teixeira JA, Oliveira R (2007) Strategies for the prevention of microbial biofilm formation on silicone rubber voice prostheses. $J$ Biomed Mat Res B 81B: 358-370.

8. Anderson JM (2001) Biological responses to materials. Annu Rev Mater Res 31: 81-110.

9. Zilberman M, Elsner JJ (2008) Antibiotic-eluting medical devices for various applications. J Cont Release 130: 202-215.

10. Gottenbos B, Grijpma D, Van der Mei HC, Feijen J, Busscher HJ (2001) Antimicrobial effects of positively charged surfaces on adhering Gram-positive and Gram negative bacteria. J Antimicrob Chemother 48: 7-13.

11. Rodrigues LR, Banat IM, Teixeira JA, Oliveira R (2006) Biosurfactants: potential applications in medicine. J Antimicrob Chem 57: 609-618.

12. Muthusamy K, Gopalakrishnan S, Ravi TK, Sivachidambaram P (2008) Biosurfactants: properties, commercial production and application. Curr Sci 94 736-747.

13. Rivardo F, Turner RJ, Allegrone G, Ceri H, Martinotti MG (2009) Anti-adhesion activity of two biosurfactants produced by Bacillus spp. prevents biofilm formation of human bacterial pathogens. Appl Microbiol Biotechnol 83: 541-553.

14. Mireles JR, Toguchi A, Harshey RM (2001) Salmonella enteric serovar Typhimurium swarming mutants with altered biofilm-forming abilities: surfactin inhibits biofilm formation. J Bacteriol 183: 5848-5854.

15. Reid G (2000) In vitro testing of Lactobacillus acidophilus NCFM as a possible probiotic for the urogenital tract. Int Dairy J 10: 415-419.

16. Boris S, Barbés C (2000) Role played by lactobacilli in controlling the population of vaginal pathogens. Microbes Infect 2: 543-546.

17. Velraeds M, Van de Belt-Gritter B, Van der Mei HC, Reid G, Busscher HJ (1998) Interference in initial adhesion of uropathogenic bacteria and yeasts to silicone rubber by a Lactobacillus acidophilus biosurfactant. J Med Microbiol 47: 1081 1085.

18. Heinemann C, Van Hylckama V, Janssen D, Busscher HJ, Van der Mei HC, et al. (2000) Purification and characterization of a surface-binding protein from Lactobacillus fermentum RC-14 that inhibits adhesion of Enterococcus faecalis 1131. FEMS Microbiol Lett 190: 177-180.

19. Rodrigues LR, Van der Mei HC, Teixeira JA, Oliveira R (2004) Biosurfactant from Lactococcus lactis 53 inhibit microbial adhesion on silicone rubber. Appl Microbiol Biotechnol 66: 306-311.

20. Rodrigues LR, Van der Mei HC, Banat I, Teixeira JA, Oliveira R (2006) Inhibition of microbial adhesion to silicone rubber treated with biosurfactant from Streptococcus thermophilus A. FEMS Immunol Med Microbiol 46: 107-112.

21. Rodrigues LR, Van der Mei HC, Teixeira JA, Oliveira R (2004) Influence of biosurfactants from probiotic bacteria on formation of biofilms on voice prostheses. Appl Environ Microbiol 70: 4408-4410.

22. Gudiña E, Rocha V, Teixeira JA, Rodrigues LR (2010) Antimicrobial and antiadhesive properties of a biosurfactant isolated from Lactobacillus paracasei subsp. paracasei A20. Lett Appl Microbiol 50:419-424.

23. Rodrigues LR, Teixeira JA, Oliveira R (2006) Low cost fermentative medium for biosurfactant production by probiotic bacteria. Biochem Eng J 32:135-142. 